\title{
Efficient Broadcasting in Ad Hoc Networks Using Directional Antennas ${ }^{\star}$
}

\author{
Fei Dai and Jie Wu \\ Department of Computer Science and Engineering, \\ Florida Atlantic University, \\ Boca Raton, FL 33431 \\ jielcse.fau.edu
}

\begin{abstract}
Using directional antennas to conserve bandwidth and energy consumption in ad hoc networks is becoming popular in recent years. However, applications of directional antennas for broadcasting have been limited. We propose a novel broadcast protocol called directional self-pruning (DSP) for ad hoc networks using directional antennas. DSP is a non-trivial generalization of an existing localized deterministic broadcast protocol using omnidirectional antennas. Compared with its omnidirectional predecessor, DSP uses about the same number of forward nodes to relay the broadcast packet, while the number of forward directions that each forward node uses in transmission is significantly reduced. With the lower broadcast redundancy, DSP is more bandwidth- and energy-efficient. DSP is based on 2-hop neighborhood information and does not rely on location or angle-of-arrival (AoA) information. DSP is a pure localized protocol. We prove that the expected number of forward nodes in DSP is within a constant factor of the minimal value in an optimal solution. Our simulation results show that DSP can reduce the transmission cost by $30 \%-65 \%$.
\end{abstract}

\section{Introduction}

Using directional antennas to conserve bandwidth and energy consumption in wireless communications is becoming popular in recent years [1]. Compared with the omnidirectional antennas, a smart antenna can form directional beams for both transmission and reception, which achieves better signal-to-noise ratio (SNR) and reduces interference. Many network protocols have been proposed for using directional antennas in ad hoc networks [2, 3, 4, 5, 6]. However, most of them focused on the MAC layer, and research on the application of directional antennas in unicasting and broadcasting has been limited.

Broadcasting is frequently used in ad hoc networks for data dissemination and ondemand route discovery. Blind flooding has high cost and excessive redundancy, which causes the broadcast storm problem [7]. Both probabilistic approaches [7] and deterministic approaches [8, 9, 10, 11, 12] have been proposed for efficient broadcasting in

\footnotetext{
* This work was supported in part by NSF grants CCR 0329741, CNS 0422762, CNS 0434533, ANI 0073736, and EIA 0130806.
} 
ad hoc networks. Probabilistic approaches need relatively high broadcast redundancy to maintain an acceptable delivery ratio. Deterministic approaches select a few forward nodes based on neighborhood information to achieve full delivery. Most deterministic broadcast schemes in ad hoc networks are localized. A localized algorithm determines the status of each node (forward or non-forward) based on its $k$-hop neighborhood information, where $k$ is a small constant.

Although deterministic algorithms are more efficient than probabilistic approaches, their broadcast redundancy is not minimized. Wireless nodes with directional antenna can control their radiation pattern to reduce broadcast redundancy. Several protocols [13, 14, 15, 16] have been proposed for efficient broadcasting using directional antennas. However, most of them are probabilistic approaches, depend on location or AoA information, and assume specific antenna models. All those protocols assume the omnidirectional reception mode. In this paper, we propose a novel broadcast protocol called directional self-pruning (DSP), which extends an omnidirectional broadcast protocol (called self-pruning) [17]. Extending the omnidirectional self-pruning scheme to use directional antennas is non-trivial. We show that the original self-pruning algorithm in [17] must be enhanced carefully to avoid broadcast failure without be overly conservative. Compared with its omnidirectional predecessor, DSP minimizes the interference and energy consumption by switching off transmission in unnecessary directions. Our simulation results show that DSP can reduce the transmission cost by $30 \%-65 \%$.

In DSP, each node is equipped with only 2-hop neighborhood information (or simply 2-hop information), which is collected via two rounds of "Hello" exchanges among neighbors. The direction information (i.e., how to form a directional beam to reach a specific neighbor) is included in the 2-hop information and does not cause extra overhead to collect. DSP uses a general antenna model with fewer assumptions than existing models. The main contributions of this paper are as follows:

1. Combine directional antennas with the latest broadcast techniques to minimize the broadcast redundancy in ad hoc networks.

2. Provide a general antenna model and a directional neighbor discovery scheme that does not rely on any location or AoA information.

3. Conduct both theoretical and simulation study to evaluate the performance of DSP.

The remainder of this paper is organized as follows. Section 2 reviews existing broadcast schemes. In Section 3, we introduce a general antenna model, a neighborhood discovery scheme, and a formal definition of the problem. Section 4 discusses the DSP algorithm and properties. Simulation results are presented in Section 5. Section 6 concludes this paper.

\section{Related Work}

Many deterministic broadcast schemes have been proposed for ad hoc networks using omnidirectional antennas. A deterministic broadcast algorithm is equivalent to an algorithm that forms a connected dominating set (CDS). The problem of finding a minimal CDS was proved NP-complete. Approximation algorithms exist, but are either centralized [18], cluster-based [19], or location-based [20]. Centralized and cluster-based 


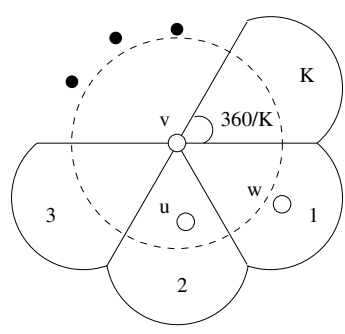

(a) ideally sectorized

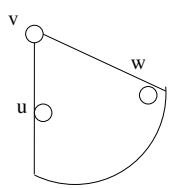

(b) adjustable cone

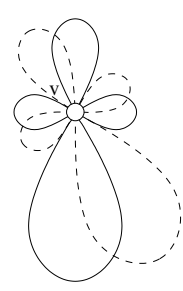

(c) irregular beam pattern

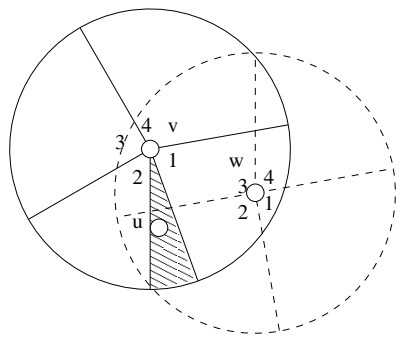

(d) a general model

Fig. 1. Directional antenna models

algorithms have slow convergency in mobile networks. Location-based algorithms rely on external devices such as GPS receivers, which cause extra cost. Localized broadcast algorithms can be further divided into neighbor designating algorithms and selfpruning algorithms. In neighbor-designating [8, 9, 11], each forward node selects a few 1-hop neighbors as new forward nodes to cover its 2-hop neighbors. In self-pruning [10, 12, 21], each node determines it own status (forward or non-forward). A generic self-pruning scheme was proposed in [17].

Application of directional antennas in localized broadcasting is limited in literature. Most of them are probabilistic approaches [14, 15, 16]. Choudhury and Vaidya [14] proposed to reduce the broadcast redundancy in relaying routing request by switching off transmissions in directions toward the last forward node. Hu, Hong, and Hou [15] presented three schemes to improve the broadcast efficiency. In the first scheme, each node switches off its transmission beams towards known forward nodes. In the second and third schemes, each forward node designates only one neighbor as a forward node in each direction. In the third scheme, the selection of forward nodes is aided by location information. Shen et al [16] devised directional versions of probabilistic protocols in [7]. Only two localized deterministic schemes were proposed [13, 16]. Lim and Kim's neighbor elimination [8] was extended in [16], where each node switches off transmission in a direction, if all neighbors in this direction are also neighbors of a known forward node. In [13], each node forms a single beam with an adjustable width to reach all neighbors that are not covered by transmissions of known forward nodes. Location information is used to calculate the angle and orientation of the transmission beam.

\section{Preliminaries}

\subsection{Antenna Model}

Two beam-forming techniques exist: switched beam and steerable beam [1]. Switched beam systems use fixed antenna patterns to transmit to or receive from specific directions. A popular antenna model for those systems is ideally sectorized [14, 15, 16], as shown in Figure 1(a). The neighborhood of each node $v$ is equally divided into $K$ nonoverlapping sectors. Each node can switch on one or several sectors for transmission or reception. Aligned sectors are assumed in most existing protocols. Steerable beam 
systems can adjust the bearing and width of a beam to transmit to or receive from certain neighbors. The corresponding antenna mode is a adjustable cone [13], as shown in Figure 1 (b). Most protocols also assume an omnidirectional mode, but the transmission range in omnidirectional mode (represented by the dashed circle in Figure 1(a)) is substantially smaller than that in directional mode. Both antenna models assume regular beam shapes. In practical systems, antenna patterns have irregular shapes due to the existence of side lobes (as shown in Figure1(c)).

This paper uses a general antenna model that does not rely on a specific beam-forming technique. As shown in Figure 1(d), each node can transmit and receive in $K$ directions with id's $1,2, \ldots, K$. The shape of each direction can be irregular, overlapping (see the shadowed area), and unaligned. Each node can transmit in one direction or several directions via sweeping [14]. The reception mode can be omnidirectional (default) or directional. For each node $v, N_{i}(v)$ denotes $v$ 's neighbor set direction $i$, and $N(v)=$ $N_{1}(v) \cup N_{2}(v) \cup \ldots \cup N_{K}(v)$ is $v$ 's complete neighbor set. A neighbor may appear in several directions when there is an overlapped area. For each neighbor $u$, its directions with respect to node $v$ is $D_{v \rightarrow u}=\left\{i \mid u \in N_{i}(v)\right\}$. For example, in Figure 1(d), $N(v)=\{u, w\}$, where $u, w \in N_{1}(v)$ and $u \in N_{2}(v)$. Therefore, $D_{v \rightarrow u}=\{1,2\}$ and $D_{v \rightarrow w}=\{1\}$. The network is viewed as a graph $G=(V, E)$, where $V$ is the set of nodes, and $E$ is the set of bidirectional links. A wireless link $(u, v) \in E$ if and only if $v \in N(u)$ and $u \in N(v)$. We assume the network is symmetric and connected via bidirectional links.

\subsection{Directional Neighborhood Discovery}

In directional neighborhood discovery, each node sends periodical "Hello" messages to its neighbors. Each "Hello" message is transmitted in all directions. By collecting "Hello" messages from its neighbors, each node $v$ can assemble its 1-hop information, including id's and directions of its neighbors. Note that the direction for $v$ to reach a neighbor $u$ is still unknown at that time. The 1-hop information is exchanged among neighbors in the next round of "Hello" messages. By assembling the 1-hop information of $v$ its neighbors, node $v$ can construct its 2-hop (direction) information, which is a subgraph of $G$ derived from $v$ 's closed neighbor set $N[v]=N(v) \cup\{v\}$, and direction $D_{u \rightarrow w}$ for any two nodes $u, v \in N[v]$. Note that $v$ 's 2-hop neighbors are excluded from the 2-hop information, because the direction from a 1-hop neighbor to any 2-hop neighbor is unknown.

In the above scheme, each "Hello" message is sent out $K$ times in $K$ directions at each node. However, given the same neighborhood area, the cost of each directional transmission is roughly $1 / K$ that of an omnidirectional transmission. The total cost of the directional neighborhood discovery is similar to that of the traditional scheme using omnidirectional "Hello" messages. This scheme also works when there are obstacles, as the neighbor and direction information is retrieved from real signal reception instead of being computed from an ideal antenna pattern. We assume that node movement, in terms of changing positions or turning on their axes, is relatively slow with respect to the "Hello" interval, so that 2-hop information collected at each node is up-to-date. We also assume that packet collision is avoided via an ideal MAC layer. For clarity, we use ideally sectorized direction shapes in examples. Nevertheless, all results in this paper work for the general antenna model as shown in Figure 1(d). 


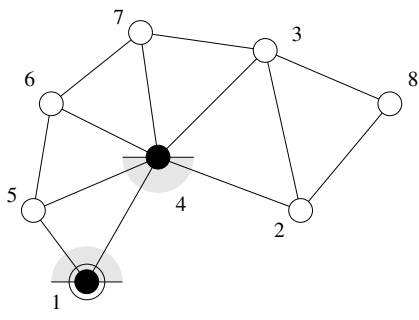

(a) a failed broadcast process

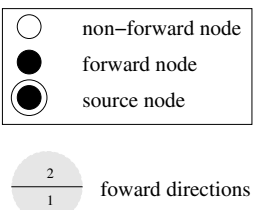

foward directions

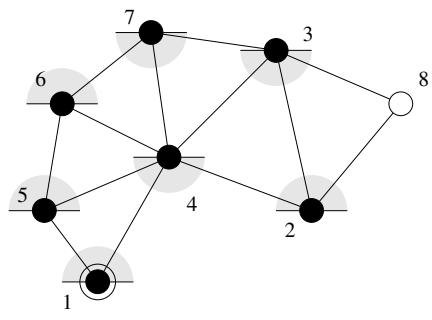

(b) an overly conservative broadcast process

Fig. 2. Problems in converting OSP to DSP

\subsection{Efficient Broadcasting}

For each broadcasting, a few forward nodes are selected to forward in some forward directions. We define the forward scheme, $F$, as a function on $V$, where $F(v)$ is the set of $v$ 's forward directions. Given $F$, we say a destination $d$ is reachable from a source $s$, if $s=d$ or there exists a forward path $P:\left(v_{1}=s, v_{2}, \ldots, v_{l}=d\right)$ satisfying that every node in $P$ forwards to the direction of its successor. A forward scheme $F$ achieves full delivery if all nodes in the network are reachable from $s$. Given an antenna model, we define the transmission cost of a forward scheme as $|F|=\sum_{v \in V}|F(v)|$.

Efficient Broadcasting. Given a number of antenna directions $K$, network $G$, and source $s$, find the forward scheme $F$ that achieves full delivery with minimum transmission cost $|F|$.

Efficient broadcasting using omnidirectional antennas is a special case of the above problem with $K=1$, which is known to be NP-complete. The efficient broadcasting problem with a particular $K \geq 2$ in a geometric graph is conjectured to be NP-complete. Our objective is to find a localized solution with a low average transmission cost.

We first review the omnidirectional self-pruning (OSP) as a trivial solution to the above problem. In OSP, each node computers the coverage of its neighborhood after receiving the packet from one or several known forward nodes. In node $v$ 's local view, a node $w$ is covered if: (1) $w$ is a known forward node, (2) $w$ is a neighbor of a known forward node, or (3) $w$ is a neighbor of a covered node with a higher id than $v$. If $v$ has uncovered neighbors, it becomes a forward node and transmits in all directions; otherwise, it does nothing. It was proved in [17] that OSP guarantees full delivery.

\section{Directional Self-pruning}

Intuitively, a forward node only needs to transmit in directions of uncovered neighbors to conserve transmission cost. However, this "optimization" is over aggressive and causes broadcast failures. As shown in Figure 2 (a), if node 4 forwards in direction 1 only, neither node 5 nor 2 will forward the packet. Nodes 3, 6, 7, and 8 will never received the packet.

A solution to the above problem is for each forward node to piggyback its forward directions to the data packet. In computing coverage, a neighbor $w$ of a forward node 


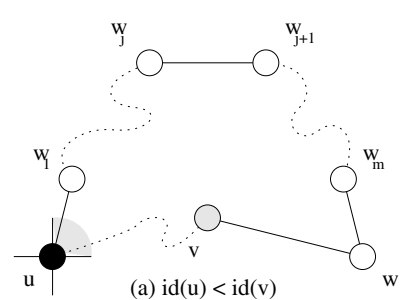

(a) $\operatorname{id}(u)<\operatorname{id}(v)$

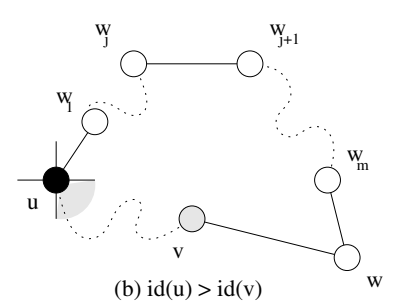

(b) id(u) $>$ id(v)

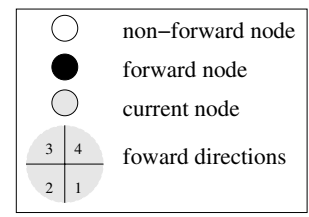

Fig. 3. Replacement paths

$u$ is not covered unless it is within a forward direction of $u$. This rule ensures full delivery, but is overly conservative and causes unnecessary transmissions. As shown in Figure 2(b), nodes 2, 3, 5, 6, and 7 become forward nodes under the new rule. However, transmissions of nodes 2 and 3 are redundant.

Directional self-pruning (DSP) uses a refined coverage rule to achieve both full delivery and high efficiency. A neighbor $w$ of node $v$ is viewed covered if and only if $w$ :

1. is a known forward node,

2. is a neighbor of a known forward node $u$ that has transmitted in $w$ 's direction, or

3 . is a neighbor of a covered node with a higher id than $v$.

As shown in Figure 3, a covered neighbor is either a forward node or connected to a forward node via a replacement path $\left(u, w_{1}, w_{2}, \ldots, w_{m}, w\right)$, where $i d\left(w_{i}\right)>i d(v)$. Two scenarios exit: (a) $i d(u)<i d(v)$, then $w_{1}$ must be within a forward direction of $u$ in order to apply term 2 ; (b) $i d(u)>i d(v)$, then $w_{1}$ can be out of $u$ 's forward directions by applying term 3 .

Based on the new rule, node 5 in Figure 2 can no longer view node 6 as covered, because node 4 has not transmitted in direction 2 and, in addition, node 4 has a lower id than node 5 . Therefore, node 5 becomes a forward node. Similarly, nodes 6,7 , and 3 become forward nodes and ensure full delivery. On the other hand, node 2 can still view nodes 3 and 8 as covered, because both nodes are connected via a replacement path to node 4 , which has a higher id than node 2 . Therefore, node 2 becomes a non-forward node.

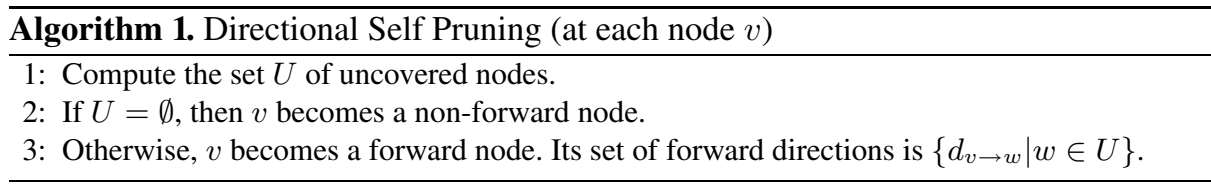

Algorithm 1 gives the DSP algorithm. For each uncovered neighbor $w$, at least one direction $d_{v \rightarrow w} \in D_{v \rightarrow w}$ must become a forward direction in $F(v)$. If directions are overlapping, each uncovered node may be within several directions (i.e., $\left|D_{v \rightarrow w}\right|>1$ ). In this case, a greedy heuristic algorithm for the set coverage problem [18] can be used to select a minimum $F(v)$ that covers all nodes in $N(v)-C$. 


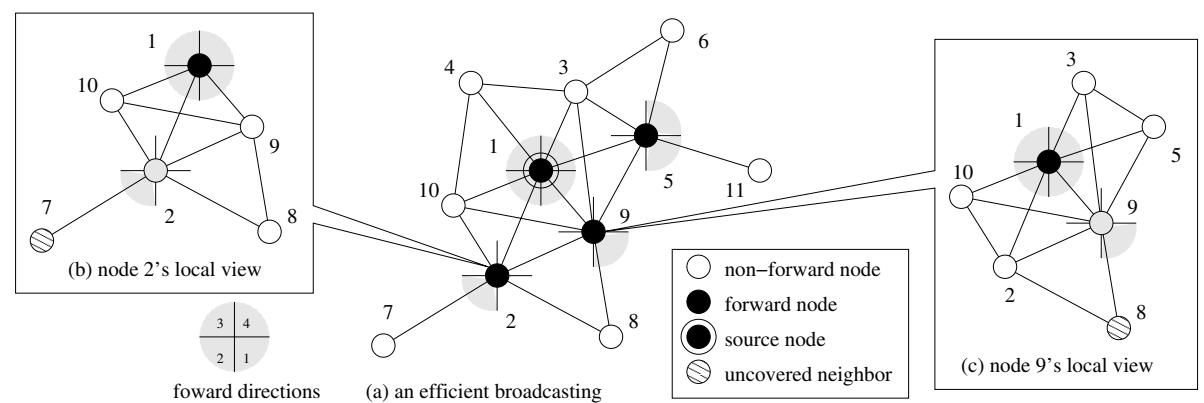

Fig. 4. Directional self-pruning

Figure 4illustrates DSP in a network with 11 nodes, each node has ideally sectorized directions with $K=4$. The source node 2 transmits in all four directions. Node 2 transmits in only one direction, because in its local view (as shown in Figure 4(b)), all neighbors except node 7 are covered. Meanwhile, there is an uncovered node 8 in node 9's local view (as shown in Figure 4(c)). Therefore, node 9 becomes a forward node and transmits in direction 1. Similarly, node 5 has two uncovered nodes 6 and 10, and transmits in directions 3 and 4. Each node receives the broadcast packet exactly once, except for the source node.

Theorem 1. The forward scheme determined by DSP achieves full delivery.

Proof. By contradiction, suppose there is at least one node that is unreachable from the source $s$. Let $U$ be the set of "border" nodes that (1) is reachable from the source node, and (2) has an unreachable neighbor. $U$ is not empty in a connected network. Let $v$ be the node with the highest id in $U$, and $w$ an unreachable neighbor of $v$. Since $v$ has not transmitted in $w$ 's direction, node $w$ must be covered in $v$ 's local view. However, we show that $w$ cannot be covered, as none of the three terms in the refined coverage rule applies:

1. $w$ is a known forward node, which implies that $w$ is reachable from $s$.

2. $w$ is a neighbor of a known forward node $u$ that has transmitted in w's direction. In this case $w$ is reachable from $s$ via a forward path through $u$.

3. $w$ is a neighbor of a covered node with a higher id than $v$. There are only two possible scenarios, as shown in Figure 3. In both cases, the unreachable node $w$ is connected to a reachable node $w_{1}$ via a path $P:\left(w_{1}, w_{2}, \ldots, w_{m}, w\right)$, where each $w_{i}(1 \leq i \leq m)$ has a higher id than $v$. There is at least one node $w_{j}$ in $P$ that has an unreachable neighbor $w_{j+1}$ (here we view $w$ as $w_{m+1}$ ). That is, $w_{j} \in U$, which contradicts the assumption that $v$ has the highest id in $U$.

Theorem 2. In random ad hoc networks, the expected number offorward nodes in DSP is $O(1)$ times that in an optimal solution.

Proof of Theorem 2 is omitted due to the limit of space. Whether a bound exists on the number of forward directions remains an open problem. 


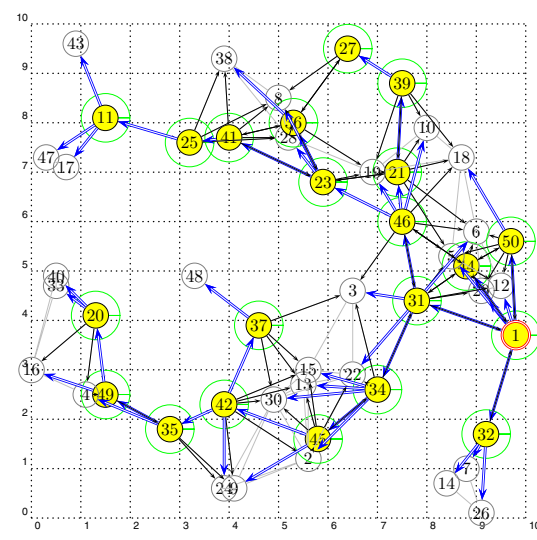

(a) OSP

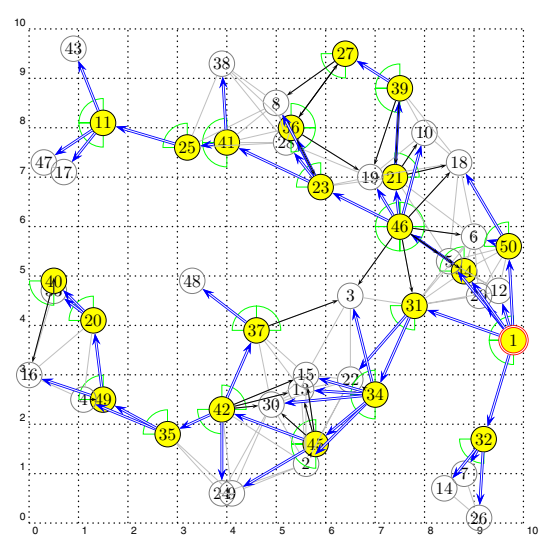

(b) $\operatorname{DSP}(K=4)$

Fig. 5. Sample broadcast processes from source node 1. Gray nodes are forward nodes and white nodes are non-forward nodes. Pies surrounding forward nodes represent forward directions. Arrows represent receptions of the broadcast packet, where double lines are first receptions, and single lines redundant receptions

\section{Simulation}

\subsection{Simulation Environment}

We simulated DSP, OSP, and blind flooding on a customized simulator. Simulations are conducted in random networks with 20-200 nodes deployed in a $100 \times 100$ area. We use two fixed ranges $r=25$ and $r=50$, which correspond to relatively sparse and dense networks, respectively. Only connected networks are used in the simulation; disconnected networks are discarded. All nodes have an ideally sectorized antenna pattern with $K$ sectors $(2 \leq K \leq 16)$. We assume no mobility or collision. The following measures are compared: (1) efficiency in terms of the number of forward nodes and normalized transmission cost $|F| / K$, (2) redundancy ratio, i.e., average number of receptions per node, and (3) average routing distance in hops. The $90 \%$ confidence intervals of these measures are within $\pm 1 \%$.

Figure 5 illustrates executions of omnidirectional and directional self-pruning algorithms in a random network with 50 nodes and an average node degree of 6 . OSP (shown in Figure 5(a)) uses 21 forward nodes. Its normalized transmission cost is also 21. Its redundant ratio is 2.74 . The average routing distances is 3.70. DSP (shown in Figure 5(b)) uses 22 forward nodes and 34 forward directions, which corresponds to a normalized transmission cost of 8.5 with 4 directions. Its redundant ratio is 1.56 . The average routing distances is 3.72 . In this example, DSP has a similar number of forward nodes and routing distance to OSP, and has lower normalized transmission cost and redundant ratio. 

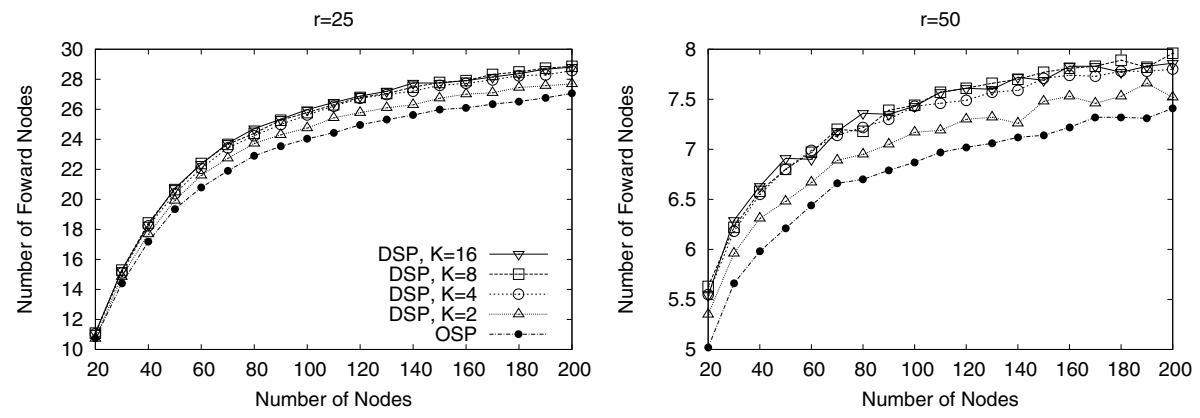

Fig. 6. Number of forward nodes
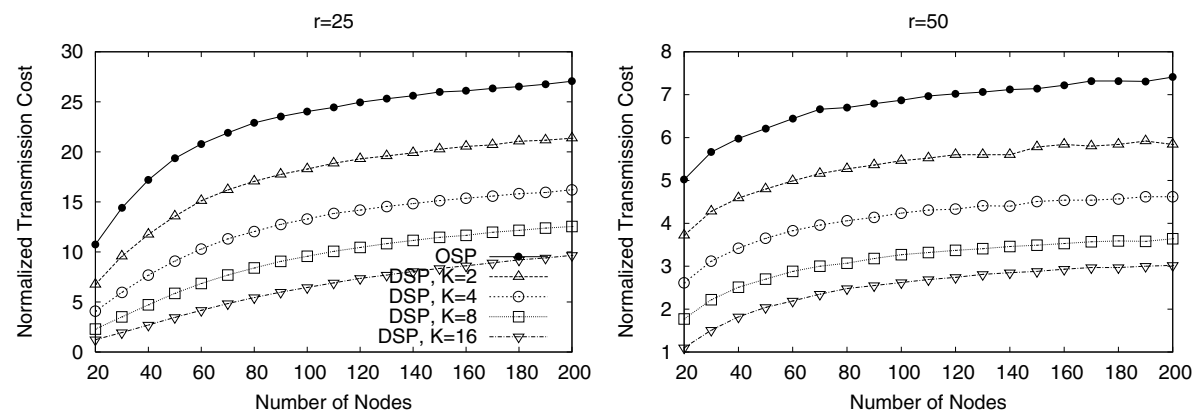

Fig. 7. Normalized transmission cost

\subsection{Simulation Results}

Efficiency. Figures 6 and 7 compare the broadcast efficiency of OSP and DSP. The left graph in Figure 6 shows the number of forward nodes in relatively sparse networks $(r=25)$, and the right graph shows results in relatively dense network $(r=50)$. The same layout is used in the following figures. For all network types, DSP uses more forward nodes than OSP. DSP uses about 5\% more forward nodes than OSP when $K=2$, and about $10 \%$ more forward nodes when $K=4,8$, or 16 . It is because fewer nodes can be marked as covered in each node's local view in DSP. It is because fewer nodes can be marked as covered in each node's local view in DSP. Based on the refined coverage rule, a neighbor $w$ of a known forward node $u$ in node $v$ 's local view may not be covered if $w$ is in a non-forward direction of $u$, and $u$ has a lower id than $v$. Using more directions may produce more uncovered nodes.

Figure 7 shows the transmission cost of different schemes. For all network types, the normalized transmission cost of DSP with $K=2,4,8$, and 16 is about $70 \%, 55 \%, 45 \%$, $35 \%$ that of OSP. The fraction of non-forward directions increases as more directions are used to create finer divisions. On the other hand, the gain in broadcast efficiency is not a linear function of $K$. Considering the complexity of forming many beam patterns, using $K=4$ or $K=8$ is good enough to conserve bandwidth and energy consumption. 

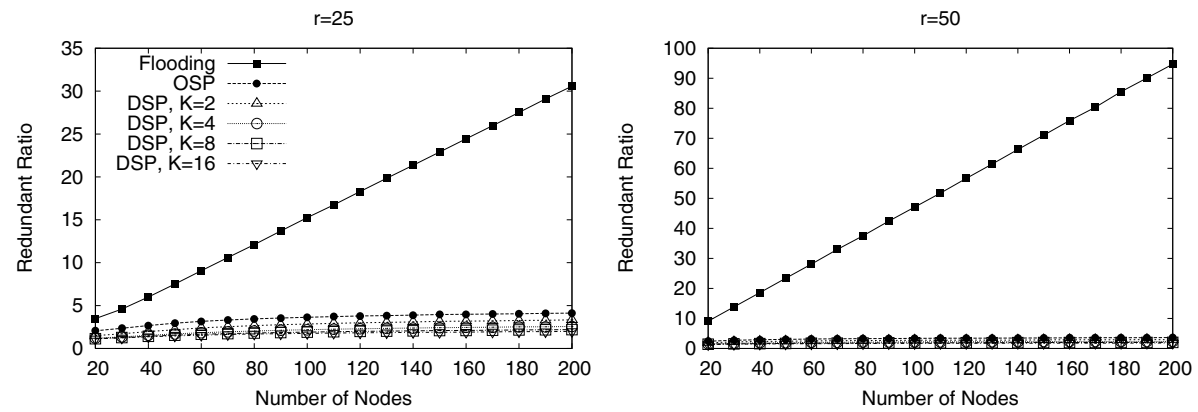

Fig. 8. Redundant ratio
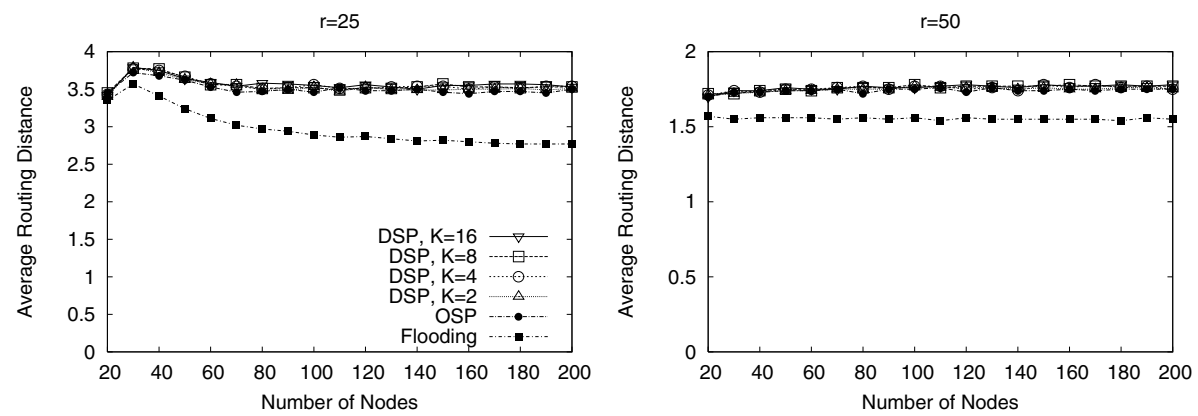

Fig. 9. Average routing distance

Redundancy. Figure 8 shows the redundancy of blind flooding, OSP, and DSP. While the redundancy ratio of blind flooding increases as the number of nodes increases, redundant ratios of the self-pruning schemes remain low. Specifically, the redundant ratio of OSP is about 4 , and that of DSP with $2 \leq K \leq 16$ is between 1.8 to 3.5. The redundancy ratio of DSP is smaller than that of OSP, but the difference is not as large as in the case of normalized transmission cost. It is because some forward nodes have "empty" directions. As DSP has very low redundancy with a larger $K$, it is very efficient in conserving the bandwidth resource. On the other hand, it may suffer a reliability problem in a real environment with packet losses caused by mobility, collision, and signal fading. In such a case, either a small $K$ or some reliability mechanisms, such as acknowledgement, should be used.

Routing distance. Figure 9 compares average routing distances. The difference between DSP and OSP is very small. Self-pruning algorithms have larger average routing distances than blind flooding. In all types of networks, the average routing distance of blind flooding is about $20 \%$ shorter than those of self-pruning algorithms. That is, if a self-pruning algorithm, omnidirectional or directional, is used to disseminate route request packets in a reactive routing protocol, the discovered route is expected to be $20 \%$ longer than the one discovered via blind flooding. In this case, tradeoffs must be done to 
balance the route discovery cost and the cost of transmitting data packets along a longer route. However, once self-pruning is selected to disseminate route request packets, using DSP will not further increase the length of the discovered route.

Simulation results can be summarized as follows: (1) DSP uses slightly more forward nodes than OSP, but has a much lower bandwidth and energy consumption. (2) The redundant ratio of DSP is 50\%-89\% that of OSP. (3) The average routing distance of DSP is very close to that of OSP, and is about $20 \%$ longer than the optimal distance.

\section{Conclusion}

We have proposed an efficient broadcast protocol for ad hoc networks using directional antennas. This protocol, called directional self-pruning (DSP), is a non-trivial generalization of an existing localized deterministic broadcast protocol using omnidirectional antennas. Compared with its omnidirectional predecessor, DSP achieves much lower broadcast redundancy and conserves bandwidth and energy consumption. DSP is based on 2-hop topology information and does not rely on any location or angle-of-arrival (AoA) information. We proved that the average number of forward nodes in DSP is within a constant factor of the minimal value in an optimal solution.

In future work, we plan to expand the proposed scheme to support neighbor designating protocols such as MPR and its variations [8, 9, 11]. Another task is the probabilistic analysis on the number of forward directions in random ad hoc networks. We expect that the average number of forward directions in DSP is also bounded as the number of forward nodes, but the technique used to calculate the number of forward nodes does not apply to the case of forward directions. We also plan to simulate DSP in more realistic networks with mobility and collisions, and embed DSP in an on-demand routing protocol to evaluate the overall routing performance.

\section{References}

1. Ramanathan, R.: On the performance of ad hoc networks with beamforming antennas. In: Proc. of ACM MobiHoc. (2001) 95-105

2. Bao, L., Garcia-Luna-Aceves, J.J.: Transmission scheduling in ad hoc networks with directional antennas. In: Proc. of ACM MobiCom. (2002) 48-58

3. Choudhury, R.R., Yang, X., Ramanathan, R., Vaidya, N.H.: Using directional antennas for medium access control in ad hoc networks. In: Proc. of ACM MobiCom. (2002) 59-70

4. Korakis, T., Jakllari, G., Tassiulas, L.: A MAC protocol for full exploitation of directional antennas in ad-hoc wireless networks. In: Proc. of ACM MobiHoc. (2003) 98-107

5. Roy, S., Saha, D., Bandyopadhyay, S., Ueda, T., Tanaka, S.: A network-aware MAC and routing protocol for effective load balancing in ad hoc wireless networks with directional antennas. In: Proc. of ACM MobiHoc. (2003)

6. Takai, M., Martin, J., Ren, A., Bagrodia, R.: Directional virtual carrier sensing for directional antennas in mobile ad hoc networks. In: Proc. of ACM MobiHoc. (2002) 183-193

7. Tseng, Y.C., Ni, S.Y., Chen, Y.S., Sheu, J.P.: The broadcast storm problem in a mobile ad hoc network. Wireless Networks 8 (2002) 153-167 
8. Lim, H., Kim, C.: Multicast tree construction and flooding in wireless ad hoc networks. In: Proc. of ACM MSWiM. (2000)

9. Lou, W., Wu, J.: On reducing broadcast redundancy in ad hoc wireless networks. IEEE Transactions on Mobile Computing 1 (2002) 111-123

10. Peng, W., Lu, X.: On the reduction of broadcast redundancy in mobile ad hoc networks. In: Proceedings of ACM MobiHoc. (2000) 129-130

11. Qayyum, A., Viennot, L., Laouiti, A.: Multipoint relaying for flooding broadcast message in mobile wireless networks. In: Proc. of HICSS. Volume 9. (2002) 298

12. Sucec, J., Marsic, I.: An efficient distributed network-wide broadcast algorithm for mobile ad hoc networks. CAIP Technical Report 248, Rutgers University (2000)

13. Cartigny, J., Simplot, D., Stojmenovic, I.: An adaptive localized scheme for energy efficient broadcasting in ad hoc networks with directional antennas. IEEE Transactions on Commuincations (2003)

14. Choudhury, R.R., Vaidya, N.H.: Ad hoc routing using directional antennas. Technical report, Dept. Electrical and Computer Engeineering, University of Illinois at Urbana Champaign (2002)

15. Hu, C., Hong, Y., Hou, J.: On mitigating the broadcast storm problem with directional antennas. In: Proc. of IEEE ICC. (2003)

16. Shen, C.C., Huang, Z., Jaikaeo, C.: Directional broadcast for ad hoc networks with percolation theory. Technical report, Computer and Information Sciences, University of Delware (2004)

17. Wu, J., Dai, F.: Broadcasting in ad hoc networks based on self-pruning. Proc. of IEEE Infocom (2003)

18. Das, B., Sivakumar, R., Bhargavan, V.: Routing in ad hoc networks using a spine. In: Proc. of IEEE IC3N. (1997) 1-20

19. Alzoubi, K.M., Wan, P.J., Frieder, O.: Distributed heuristics for connected dominating sets in wireless ad hoc networks. Journal of Communications and Networks 4 (2002) 22-29

20. Liao, W.H., Tseng, Y.C., Sheu, J.P.: GRID: A fully location-aware routing protocol for mobile ad hoc networks. Telecommunication Systems 18 (2001) 37-60

21. Dai, F., Wu, J.: An extended localized algorithm for connected dominating set formation in ad hoc wireless networks. IEEE Transactions on Parallel and Distributed Systems 15 (2004) 902-920

22. Williams, B., Camp, T.: Comparison of broadcasting techniques for mobile ad hoc networks. In: Proceedings of MobiHoc. (2002) 194-205 